\title{
Basophilic Stippling Measurement
}

National Cancer Institute

\section{Source}

National Cancer Institute. Basophilic Stippling Measurement. NCI Thesaurus. Code C96567.

The determination of the amount of basophilic stippling present in a sample. 